\title{
ARTICLE
}

\section{Faden operation in consecutive esotropia}

\author{
Min-Jung Kim ${ }^{1} \cdot$ Kun-Hoo $\mathrm{Na} \mathbb{( 1}^{1} \cdot$ Seung-Hyun Kim ${ }^{1}$
}

Received: 13 February 2019 / Revised: 17 April 2019 / Accepted: 29 April 2019 / Published online: 9 May 2019

(c) The Royal College of Ophthalmologists 2019

\begin{abstract}
Purpose To evaluate the efficacy of Faden procedure for correcting consecutive esotropia (ET).

Methods This retrospective study included 25 children who developed consecutive ET after primary bilateral lateral rectus (BLR) recession and underwent medial rectus (MR) recession with the Faden procedure (Faden group) or MR recession only (control group) between 2013 and 2018. Postoperative deviation angles were evaluated at each follow-ups until postoperative 6-month visit. Surgical motor and sensory outcomes were compared between Faden group and control group.

Results There were 10 children in the Faden group and 15 children in the control group. While the Faden group maintained orthotropia without any small deviation until postoperative 6-month visit, the control group showed wider distribution of postoperative deviation angles ( 1 patient with small angle esodeviation $<5 \mathrm{PD}, 3$ patients with esodeviation $>5 \mathrm{PD}$, and 3 patients with exodeviation $<5$ PD). In the Faden group, seven patients have good stereopsis ( $60^{\prime \prime}$ or better) and three patients demonstrated fair stereopsis (80-3000") after surgery. In the control group, four, eight, and three patients showed good, fair, and nil stereopsis $(P=0.026)$, respectively.
\end{abstract}

Conclusion MR recession combined with Faden operation could be a good surgical option for managing consecutive ET.

\section{Introduction}

Consecutive esotropia (ET) can cause persisting or variable esodeviation after surgery for exotropia despite patching and prism glasses. The exact cause of consecutive ET remains unclear. Consecutive ET may be induced by relatively more innervational input to the medial rectus (MR) muscle after bilateral lateral rectus (BLR) recession which increases MR muscle tone [1]. Strong and variable tonic convergence or tight MR muscle might be important in the development of consecutive esotropia [1,2].

Faden operation, also called posterior fixation suture, can be used to weaken the rotational force of a rectus muscle when the eye rotates towards the muscle. The arc of contact of the MR muscle can be significantly lengthened by 12-14 $\mathrm{mm}$ with the Faden operation [3]. It has been proposed that the Faden operation can specifically treat tonic esodeviation [4].

Seung-Hyun Kim

ansaneye@hanmail.net

1 Department of Ophthalmology, Korea University College of Medicine, Seoul, Korea
To the best of our knowledge, the Faden operation in consecutive esotropia was only mentioned as a case report in a text book [5]. No previous study has addressed the effect of the Faden procedure on surgical outcomes in consecutive ET. Therefore, the objective of this study was to evaluate motor and sensory outcomes in consecutive ET patients undergoing MR recession with or without the Faden procedure.

\section{Methods}

\section{Subjects}

This study was approved by the Institutional Review Board of Korea University Medical Center. It adhered to tenets of the Declaration of Helsinki. We identified patients who underwent surgical treatment for consecutive ET after BLR recession between January 2013 and December 2018 and reviewed their electronic medical records. Patients were included if they received MR recession for the treatment of consecutive ET. We excluded patients with anterior/posterior segment pathology, concomitant cyclovertical muscle surgery, older than 15 years at the time of consecutive ET surgery, or follow-up of less than 6 months after consecutive ET surgery. 


\section{Preoperative assessment}

Preoperative ophthalmologic assessment included measurement of the deviation angle by prism and alternate cover testing at $1 / 3 \mathrm{~m}$ and $6 \mathrm{~m}$, motor functions such as version and duction, the presence of dissociated vertical deviation (DVD) and inferior oblique overaction (IOOA), and fundus examinations. Refractive errors by cycloplegic refraction were evaluated as spherical equivalent values.

\section{Indication for surgery and intraoperative procedures}

Surgery was indicated when esodeviation increased over 15 PD despite non-surgical management including strict alternating occlusion and base-out prisms. All patients underwent unilateral or bilateral MR recession under general anesthesia by the same surgeon (SHK) who had combined the Faden operation with MR recession since 2016. Therefore, patients with consecutive ET who underwent a MR recession with the the Faden procedure during 2016-2018 were assigned into the Faden group while those who underwent MR recession only during 2013-2015 were assigned into the control group. Surgical dose of MR recession was determined based on the near deviation angle. Bilateral MR recession was considered for esodeviation of 30 PD or greater (Table 1). For unilateral surgery, the Faden procedure was combined with MR recession. For cases undergoing bilateral MR recession, the Faden procedure was chiefly performed in the deviating eye. All surgical procedures were performed through an inferonasal fornix incision. During the Faden procedure with MR recession, once the MR was removed from the original insertion, the posterior fixation suture was placed through the sclera 12 $\mathrm{mm}$ posterior to the MR insertion in the center of the arc of contact using a non-absorbable suture on a spatula needle. The MR muscle was reattached to the sclera by two 6-0 polyglactin 910 sutures.

Table 1 Surgical dosage of medial rectus muscle recession

\begin{tabular}{lll}
\hline $\begin{array}{l}\text { Prism } \\
\text { diopters }\end{array}$ & Unilateral recession $(\mathrm{mm})$ & Bilateral recession $(\mathrm{mm})$ \\
\hline 15 & 5.0 & \\
20 & 6.0 & \\
25 & 6.5 & \\
30 & & $4.0 / 5.0$ \\
35 & & $5.0 / 5.0$ \\
40 & & $5.0 / 6.0$ \\
45 & & $6.0 / 6.0$ \\
50 & & $6.5 / 6.5$ \\
\hline
\end{tabular}

\section{Postoperative measurements}

All patients were followed up postoperatively at 1 day, 1 week, 1 month, 3 months, and 6 months. At each visit, we evaluated deviation angle, version, and duction. We also assessed stereoacuity using the Titmus Test (Stereo Optical Co. Inc., Chicago, IL, USA) at $1 / 3 \mathrm{~m}$. Distant fusion was assessed with Vectogram (Luneau L29 chart projector; Luneau SAS, Chartres, France) at $6 \mathrm{~m}$.

\section{Outcomes and statistical analysis}

Baseline ophthalmologic findings were compared between the Faden group and the control group. We calculated a mean deviation angle with a standard deviation (SD) at postoperative 1 day, 1 week, 1 month, 3 months, and 6 months. Surgical motor and sensory outcomes were evaluated at postoperative 6-month visit according to groups. We classified motor outcomes into orthotropia without any small deviation, eso-/exodeviation $<5 \mathrm{PD}$, and eso/exodeviation $\geq 5 \mathrm{PD}$. Results of stereoacuity test were divided into good (60" or better), fair $\left(80-3000^{\prime \prime}\right)$, or nil. For comparison between groups, stereoacuity numbers were changed into the logarithm of their values [6]. Results of Vectogram were either fusion or suppression. Statistical analyses were conducted using SPSS for Windows (V.18.0; SPSS Inc, Chicago, IL, USA). Mann-Whitney $U$ test was used to compare continuous variables. Associations between categorical variables were evaluated using Fisher's exact test.

\section{Results}

Thirty-three patients underwent MR recession for consecutive ET after BLR recession during the study period. They were initially enrolled in this study. However, eight patients were excluded due to the following reasons: previous cataract surgery (one patient), concomitant cyclovertical muscle surgery (two patients), older than 15 years at the time of consecutive ET surgery (two patients), and observation period of less than 6 months (one patient). Therefore, 25 patients were included in this study, including 10 in the Faden group and 15 in the control group. Baseline characteristics including age at primary BLR recession, age at consecutive ET surgery, preoperative angles of deviation, and refractive errors were comparable between the two groups (Table 2).

The Faden group maintained orthotropia without any small deviation from postoperative 1 week until 6-month visit, making the SD of deviation angles being zero. The control group demonstrated a mean esodeviation of less than 5 PD during the follow-up period, showing no 
Table 2 Characteristics of patients with consecutive esotropia who underwent medial rectus muscle recession with or without the faden procedure

\begin{tabular}{llll}
\hline & Faden group $(n=10)$ & Control $(n=15)$ & $P$-value \\
\hline Female sex $(n, \%)$ & $6(60 \%)$ & $10(66.7 \%)$ & $1.000^{\mathrm{a}}$ \\
Age at primary BLR recession (years) & $6.8 \pm 1.8(3.8-10.0)$ & $5.3 \pm 2.7(1.2-8.8)$ & $0.177^{\mathrm{b}}$ \\
Age at surgery for consecutive esotropia & $8.4 \pm 1.2(6.9-10.5)$ & $7.1 \pm 2.1(3.3-10.3)$ & $0.080^{\mathrm{b}}$ \\
(years) & & & \\
Preoperative esodeviation (prism diopters) & & & \\
$\quad$ Near & $29.7 \pm 13.8(16-55)$ & $24.7 \pm 11.1(15-45)$ & $0.176^{\mathrm{b}}$ \\
$\quad$ Distant & $29.2 \pm 14.0(16-55)$ & $24.0 \pm 10.5(15-45)$ & $0.179^{\mathrm{b}}$ \\
DVD associated & $0(0 \%)$ & $2(13.3 \%)$ & $0.500^{\mathrm{a}}$ \\
IOOA associated & $1(10 \%)$ & $2(13.3 \%)$ & $1.000^{\mathrm{a}}$ \\
Refractive errors (diopters) & $-1.3 \pm 1.4(-3.4$ to 0.6$)$ & $-0.8 \pm 1.4(-3.0$ to 2.3$)$ & $0.653^{\mathrm{b}}$ \\
Bilateral MR recession $(n, \%)$ & $4(40 \%)$ & $6(60 \%)$ & $1.000^{\mathrm{a}}$ \\
\hline
\end{tabular}

$B L R$ bilateral lateral rectus, $D V D$ dissociated vertical deviation, IOOA inferior oblique overaction, $M R$ medial rectus, $N A$ not applicable

${ }^{\mathrm{a}}$ Fisher exact test

${ }^{\mathrm{b}}$ Mann-Whitney $U$ test
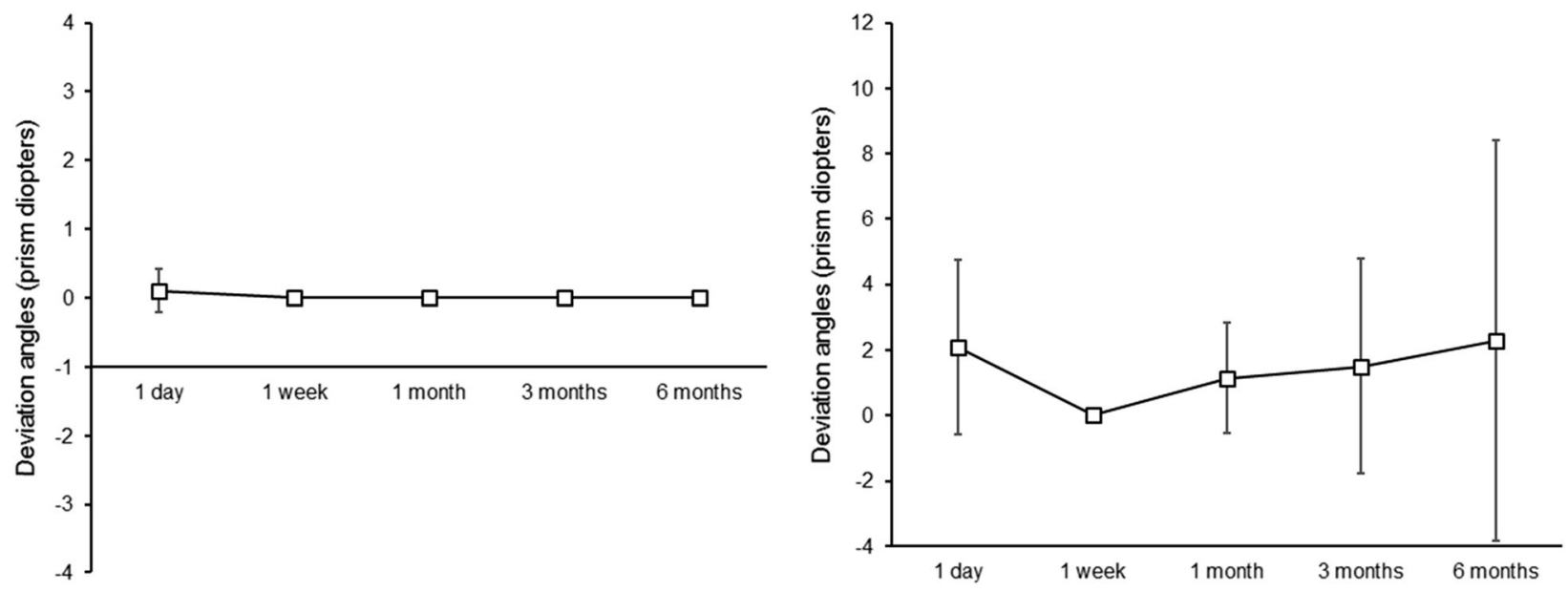

Fig. 1 Mean deviation angles of the Faden group (Left) and the control group (Right) at postoperative follow ups. Plus values mean esodeviation

significant difference compared to the Faden group at each time point. However, its greater SD value represented wider distribution of postoperative deviation angle (Fig. 1). At postoperative 6-month visit, all patients in the Faden group showed orthotropia. In the control group, 1 patient had esodeviation of $2 \mathrm{PD}, 3$ patients demonstrated $6 \mathrm{PD}, 10 \mathrm{PD}$, and $20 \mathrm{PD}$ of esodeviation, and 3 patients demonstrated 2 PD, 4 PD, and 4 PD of exodeviation, respectively (Table 3). Limitation on version or duction was not observed in either group.

In the Faden group, seven patients had good stereopsis $\left(60^{\prime \prime}\right.$ or better) while three patients demonstrated fair stereopsis. In the control group, four, eight, and three had good, fair, and nil stereopsis $(P=0.026)$, respectively. All patients in the Faden group demonstrated distant fusion while $46.7 \%$ of the control group showed suppression $(P=$ $0.020)$.

\section{Discussion}

There is no consensus on the pathogenic convergent mechanism of consecutive ET. The tonic part of convergence varies with time. It is responsible for the variance of esodeviation [7]. We hypothesized that tonic esodeviation plays a major role in the development of consecutive esotropia after BLR recession. Following BLR recession, MR recession can typically be performed if there is no significant limitation of abduction $[8,9]$.

In this study, MR recession and additional posterior fixation suture showed excellent surgical results in all ten cases with consecutive ET. Conventional MR recession might be insufficient, resulting in some degree of deviation. In this situation, when Faden operation is combined with MR recession, the effect of recession is augmented since posterior fixation suture adds an additional weakening effect 
Table 3 Surgical outcomes in the two groups of consecutive esotropia according to the surgical procedures at postoperative 6 months

\begin{tabular}{|c|c|c|c|}
\hline & $\begin{array}{l}\text { Faden group } \\
(n=10)\end{array}$ & $\begin{array}{l}\text { Control } \\
(n=15)\end{array}$ & $P$-value \\
\hline \multicolumn{4}{|l|}{ Motor outcomes } \\
\hline Orthotropia & $10(100 \%)$ & $8(53.3 \%)$ & $0.020^{\mathrm{a}}$ \\
\hline $\begin{array}{l}\text { Exodeviation }<5 \\
\text { prism diopters }\end{array}$ & $0(0 \%)$ & $3(20.0 \%)$ & \\
\hline $\begin{array}{l}\text { Esodeviation }<5 \\
\text { prism diopters }\end{array}$ & $0(0 \%)$ & $1(6.7 \%)$ & \\
\hline $\begin{array}{l}\text { Exodeviation } \geq 5 \\
\text { prism diopters }\end{array}$ & $0(0 \%)$ & $0(0 \%)$ & \\
\hline $\begin{array}{l}\text { Esodeviation } \geq 5 \\
\text { prism diopters }\end{array}$ & $0(0 \%)$ & $3(20.0 \%)$ & \\
\hline \multicolumn{4}{|l|}{ Sensory outcomes } \\
\hline \multicolumn{4}{|l|}{ Stereoacuity } \\
\hline $60^{\prime \prime}$ or better & $7(70 \%)$ & $4(26.7 \%)$ & $0.026^{\mathrm{b}}$ \\
\hline $80-3000^{\prime \prime}$ & $3(30 \%)$ & $8(53.3 \%)$ & \\
\hline Nil & $0(0 \%)$ & $3(20.0 \%)$ & \\
\hline Distant suppression & $0(0 \%)$ & $7(46.7 \%)$ & $0.020^{\mathrm{a}}$ \\
\hline
\end{tabular}

${ }^{\mathrm{a}}$ Fisher exact test

${ }^{b}$ Mann-Whitney $U$ test. The results of stereopsis was analyzed by changing the stereoacuity numbers to the logarithm of their values

without significantly increasing the limitation of movement [3]. The effect of MR recession is enhanced because all slackness is taken up in the relatively short portion of the muscle between its anatomical and new insertion of the posterior fixation suture $[3,10]$. In addition, all patients in the Faden group maintained orthotropia until postoperative 6-month visit. The Faden operation can also achieve posterior stabilization of rectus muscle position [3]. We believe that MR recession combined with Faden operation can be appropriate for patients with consecutive ET who have strong and variable tonic convergence.

Faden procedures can be complicated by difficult intrasurgical access, muscle necrosis secondary to tight sutures, limitation of adduction and no effect on angle of deviation $[11,12]$. Although patients enrolled in this study did not have such problems associated with the Faden procedure, clinicians should consider both sides of the Faden procedure.

Our study has several limitations. Firstly, this is a preliminary study with a small sample size and short observation period. Moreover, as the classification of patients into the two surgical groups was based on certain times when the surgeon introduced the Faden procedure, such non-randomized methods for grouping would be prone to bias. Larger studies with longer follow-up periods would be necessary to confirm the findings obtained by our study. Secondly, we did not compare the outcomes of our study with other options such as combined resection/recession procedure.

In conclusion, the Faden operation on the MR muscle can yield additional weakening of convergence. Thus, MR recession in addition to the Faden operation can be a good surgical option for consecutive ET after BLR recession.

\section{Summary}

\section{What was known before}

- Usually MR recession would be done in consecutive esotropia which may develop due to strong tonic convergence or tight MR muscle.

\section{What this study adds}

- Faden operation associated with MR recession on the MR muscle can produce much stable outcome and yield additional weakening of convergence.

\section{Compliance with ethical standards}

Conflict of interest The authors declare that they have no conflict of interest.

Publisher's note: Springer Nature remains neutral with regard to jurisdictional claims in published maps and institutional affiliations.

\section{References}

1. Cho YA, Kim SH. Role of the equator in the early overcorrection of intermittent exotropia. J Pedia Ophthalmol Strabismus. 2009;46:30-4.

2. Carlson MR, Jampolsky A. Lateral incomitancy in intermittent exotropia: cause and surgical therapy. Arch Ophthalmol. 1979;97:1922-5.

3. Harcourt B. Faden operation (posterior fixation sutures). Eye. 1988;2:36-40.

4. Thouvenin DA, Sotiropoulos MC, Arne JL, Fournie PR. Esotropias that totally resolve under general anesthesia treated exclusively with bilateral fadenoperation. Strabismus. 2008;16:131-8.

5. Rosenbaum AL, Santiago AP. Infantile exotropia. In: Kraft SP, editors. Clinical strabismus management (Principles and Surgical techniques). Philadelphia: W.B. Saunders; 1999. p. 173. Chapter 12 .

6. Peyman A, Peyman M, Akhlaghi M. Correct method for statistical analysis of stereopsis in ophthalmology research. Graefes Arch Clin Exp Ophthalmol. 2012;250:781. author reply 783.

7. Lee TE, Kim SH. Accommodative and tonic convergence and anatomical contracture in partially accommodative and nonaccommodative esotropia. Ophthalmic Physiol Opt. 2012;32:535-8. 
8. Helveston Em. Surgical management of strabismus. In: Heveston Em An atlas of strabismus surgery: comprehensive management of motility disorders, 4th ed. St. Louis: CV Mosby; 1993. p. 448-9.

9. Plager DA. Strabismus surgery: basic and advanced strategies. New York: Oxford University Press; 2004. p. 27-8.

10. Cüppers $\mathrm{C}$, editor The so-called fadenoperation: surgical corrections by small-defined changes of the arc of contact. The 2nd
Congress of the International Strabismology Association Diffusion Generale de Librairie. Marseilles, France; 1976.

11. Wright KW. Color Atlas of strabismus surgery: strategies and techniques. 3rd ed. New York: Springer; 2007. p. 204.

12. Paliaga GP, Braga M. Passive limitation of adduction after Cuppers "faden operation" on medial recti. $\mathrm{Br} \mathrm{J}$ Ophthalmol. 1989;73:633-5. 